Classification

Physics Abstracts

42.30. ${ }^{-} \mathrm{d}$

\title{
On the way to a multi-task near field optical microscope: Simultaneous STM/SNOM and PSTM imaging
}

\author{
Maria Garcia-Parajo, Jean Rosiu and Chen Yong \\ Laboratoire de Microstructures et de Micro-électronique, CNRS, BP. 107, 196 Avenue Henri Ravera, \\ 92225 Bagneux, France
}

(Received July 4; accepted October 26, 1994)

\begin{abstract}
Résumé. - Un microscope à effet tunnel (STM) peut être utilisé en même temps comme un microscope optique en champ proche (SNOM), si la pointe du STM est remplacée par une pointe en fibre optique monomode métallisée avec de l'or, et si l'on ajoute un montage optique pour l'excitation et la détection. Grâce à une meilleure régulation de distance entre la pointe et l'échantillon, et surtout à une très courte distance de régulation $(<1 \mathrm{~nm})$ obtenue à l'aide de l'asservissement du courant tunnel, la résolution du microscope optique en champ proche est fortement augmentée. Nous décrivons notre système STM/SNOM combiné, réalisé à partir d'un microscope STM commercial fabriqué par Park Scientific Instrument, en précisant deux configurations d'excitation différentes: l'excitation en transmission ou par diffusion. Des images obtenues simultanément par les canaux du STM et du SNOM en mode collection sont comparées et interprétées. Nous présentons également nos premières images PSTM obtenues avec un microscope multitâche (AFM, STM, PSTM et SNOM) mis au point au laboratoire à partir d'un microscope optique Zeiss. Enfin, nous évoquons de nouvelles perspectives d'application concernant ce microscope multitâche.
\end{abstract}

\begin{abstract}
A scanning tunneling microscope (STM) can be also used as a scanning near-field optical microscope (SNOM) if the conventional STM tip is replaced by a gold coated monomode optical fibre point, and an excitation-collection optical set-up is added to the system. Due to the excellent control of tip-sample separation and especially, to the short gap separation between tip and sample $(<1 \mathrm{~nm})$ controlled by the tunneling current, large improvements on the optical resolution and contrast are obtained. We describe the combined STM/SNOM system, based on a commercial STM microscope from Park Scientific Instruments, using two different excitation configurations: transmission and scattering. Simultaneous images obtained by STM and collection mode-SNOM are compared and discussed. We also describe a home-made multi-task microscope (AFM, STM, PSTM, SNOM) based on a Zeiss optical microscope and present our first tests on PSTM imaging. Finally, we mention new perspectives and applications of the developed technique.
\end{abstract}

\section{Introduction.}

Much interest has been generated over the last few years into the development of near field optical microscopy for its ability to combine simultaneously the advantages of both traditional (far-field) optical microscopy and high resolution (electron-beam and scanned probes) microscopy. Scanning near-field optical microscopy (SNOM) involves illuminating a sample with a spot of light of 
sub wavelength dimensions. Generally, this is achieved by passing light through a small aperture and positioning the aperture close enough to the surface to be scanned (within the near field) so that no diffraction takes place [1-3]. In this case, the aperture works as an illuminator, with the light transmitted through the sample being collected in the far field by an objective [2-5]. Similarly, in a collection mode-SNOM [6], light from the far field is used to illuminate the sample, and the collection is made in the near field by the aperture. In both cases -illumination or collection modes- the spatial resolution obtained is far superior than that of conventional optical microscopy, i.e., if the aperture is in close proximity to the object to be resolved, then the resolution is determined primarily by the size of the aperture rather than the wavelength, $\lambda$.

As can be understood, a good achievement in near field optical microscopy depends on a large extent on the tip to sample separation, and on the tip fabrication for imaging. Several different mechanisms for distance regulation have been proposed to SNOM, being electron-tunneling [7, $8]$ and shear force sensing $[9,10]$ the more accepted ones. Electron tunneling distance-regulation (base of scanning tunneling microscopy, STM) offers an inherent advantage over other techniques because of its excellent control of tip-sample separation. In practice however, the fabrication of high quality optical probes being simultaneously conductive, is a hard task. Recently, Lieberman et al. [8] have reported on simultaneous STM and SNOM imaging using a metal coated micropipette in the illumination mode. Alternatively, we have demonstrated a new approach to the combined STM/SNOM microscopy using directly a single-mode optical fibre ending in a fine metallic point [11]. We have used a standard STM equipment for the regulation of the tip to sample separation and also for the acquisition of the STM image. The conventional STM metallic point was replaced by a gold coated optical fibre point and an excitation-detection optical set-up was added to complete the instrument. We believe that in our scheme two main factors contribute to the improvement of the lateral optical resolution and contrast of our images: (a) the short and reliable gap separation controlled by the tunneling current, and (b) the optimisation of the fabrication process for the gold coated fibre probes. In this paper we present some new results of simultaneous STM and SNOM images in the collection mode with samples excited from the far field within both transmission and scattering configurations. We also describe our last advances on the development of a multi-task system (STM, AFM, PSTM, SNOM) based on a Zeiss optical microscope.

\section{Experimental set-up.}

2.1 USING A COMMERCIAL STM EQUIPMENT. - Our approach is schematically described in figure 1. It involves basically a commercial STM equipment (Park Scientific Instrument [12]) and a branch for the optical excitation-detection. Details of the experimental procedure can be found somewhere else [11]. Since the optical fibre point is coated with gold, the collection of the STM signal and tunneling regulation is performed essentially in the standard STM way. On the other hand, under our configuration, the sample has been excited in two different ways, i.e., transmission, where the sample is illuminated from the back, and scattering, where the sample is illuminated from the top. Light transmitted (scattered) through (off) the sample is collected by the fibre point and guided to the photomultiplier. The resultant signal is amplified, detected by the lock-in amplifier and finally fed to the second channel of the STM module.

As mentioned before, one of the most difficult tasks in our combined STM/SNOM microscope is the fabrication of high quality probes. The crucial optical role of the tip morphology and especially its final radius of curvature makes any decrease of the latter and better control of the former very desirable. Moreover, if in fact a small aperture is required for high resolution, it is also true that the small intensity of the collected or transmitted light from a narrow aperture places 


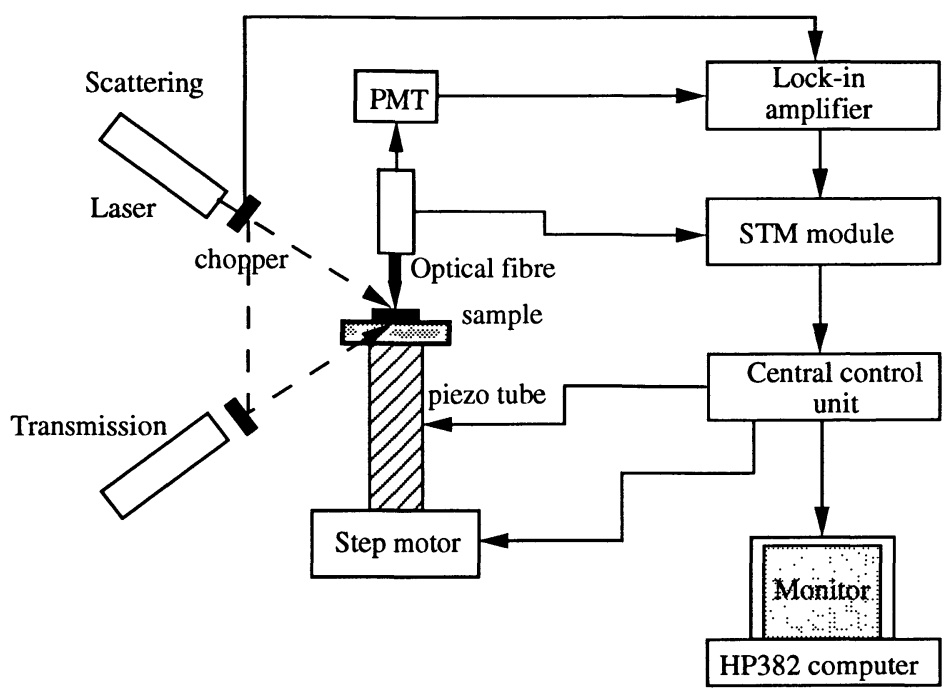

Fig. 1. - Schematic diagram of the set-up for simultaneous STM and collection mode-SNOM using a gold coated optical fibre probe.

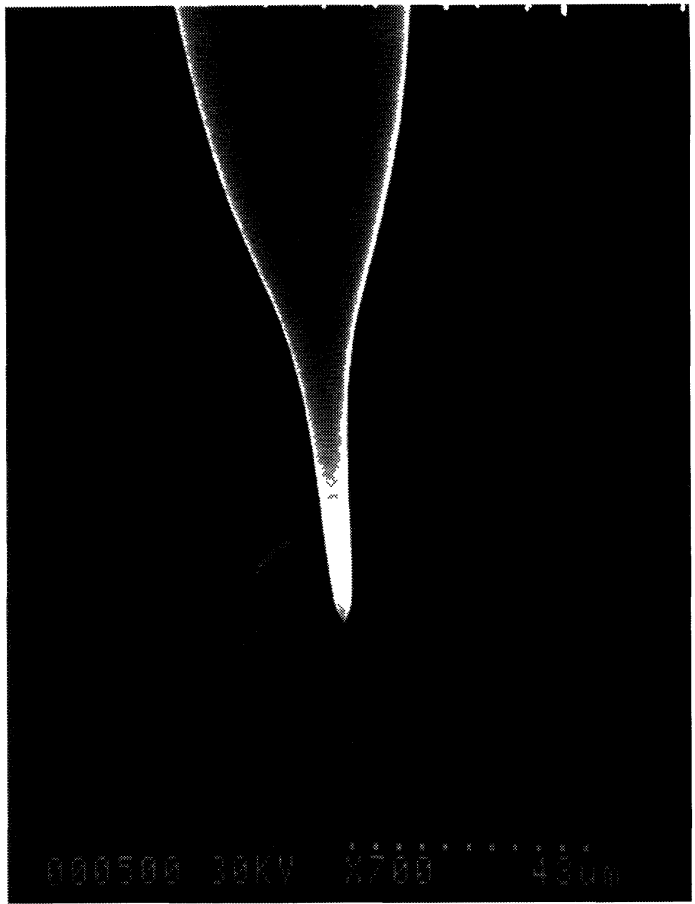

a)

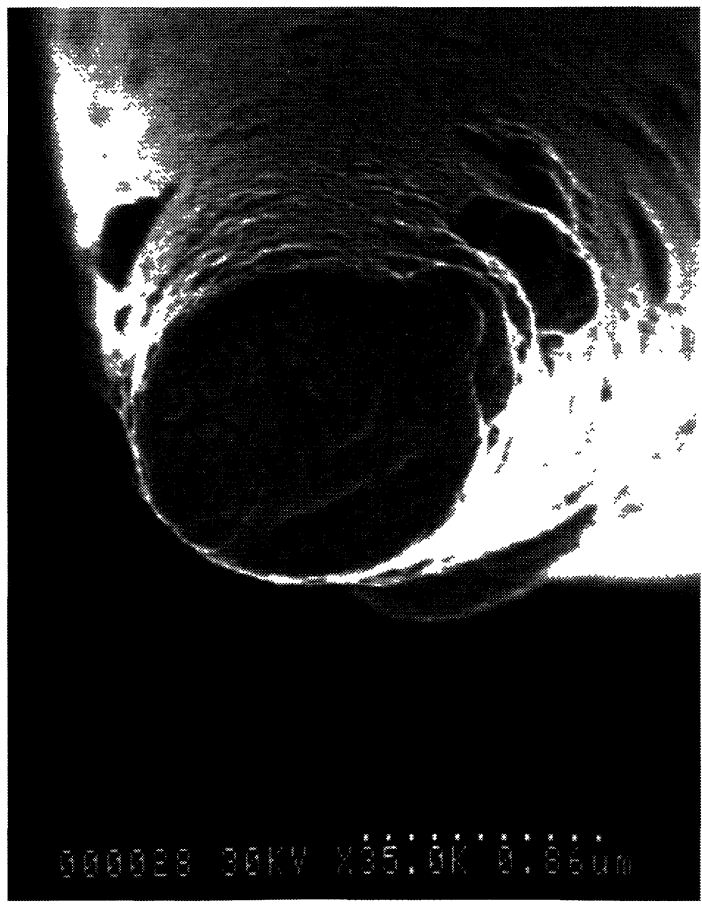

b)

Fig. 2. - SEM micrographs of a gold coated optical fibre point (a), and an enlargement of the tip region (b). 
a limit on the useful tip diameter. This optimum diameter is therefore the result of a trade-off between resolution and signal-acquisition time. Additionally, to make the point useful for tunneling regulation, it has to be coated with metal, but still with an opened aperture at the end. For the fabrication of the points we have used the commonly heating \& pulling method and finally we coated them with gold using angled evaporation. The precise control of the parameters on the puller have allowed us to obtain tip diameters down to $30 \mathrm{~nm}$. Large intensity is also obtained by careful control of the tip morphology and by the addition of the metallic coating which helps to confine the light inside the fibre [13]. Figure 2 shows an SEM photograph of the optimised probe used on the experiments with an enlargement of the tip region.

The calibration samples used for testing the resolution and the performance of the microscope were fabricated by e-beam lithography and standard wet etching process [11]. The samples consisted on a series of gratings of different diameters fabricated on $\mathrm{SiO}_{2}$ substrates. Sample 1 contained $\mathrm{Cr} / \mathrm{SiO}_{2}$ gratings with periods going from $1 \mu \mathrm{m}$ down to $200 \mathrm{~nm}$ and height of $50 \mathrm{~nm}$. Sample 2 contained $\mathrm{Al} / \mathrm{Ge} / \mathrm{SiO}_{2}$ gratings of the same period and height. A thin layer of gold $(10 \mathrm{~nm})$ was uniformly evaporated onto both samples to ensure the good electrical conductivity necessary for distance regulation, and still being semi-transparent for the optical measurements.

2.2 DEVELOPMENT OF A MULTI-TASK SYSTEM. - One of our major goals is to construct a multitask microscope able to perform PSTM and SNOM measurements using in the latter case either STM or shear force sensing as the tip-to-sample regulation mechanisms. With that aim, we have built a new system based on a Zeiss optical microscope. Figure 3a shows the schematic of the adapted microscope. An $X, Y$ translation stage sits on the base, and integrated to it is the piezo $-10 \mu \mathrm{m}$ scan rate for $X, Y$ directions, and $2.5 \mu \mathrm{m}$ in the $Z$ direction- and the sample support. The step motor needed for the vertical approach of the sample to the fix probe point (in the upper part of the microscope) is mechanically coupled to the focusing knob of the microscope. Two different probe supports have been built and fixed in the upper part of the microscope (replacing the objectives). One of the probe supports allows the positioning of the metallic tip for STM measurements on its own, or the metallic optical fibre for simultaneous STM/SNOM or pure PSTM measurements. The second probe support allows the insertion of a dither piezo together with the fibre point for shear force regulation. In this way, it is sufficient to rotate the objective head to have either STM or shear force regulation for the optical imaging.

We have also built all the appropriate circuits and electronic interfaces necessary to control the instrument. Hence, for STM regulation and imaging (Fig. 3b) an small current to voltage preamplifier was built and placed in one of the objective supports, so that the point and the amplifier are very close to each other to minimise noise. In the case of PSTM measurements (Fig. 3c), an equivalent circuit was built to amplify the signal coming from the PMT before being sent to the STM module. Both amplifiers, together with the step motor and piezo are controlled by the central control unit in the same way as is done in the commercial STM instrument, through an electronic interface.

\section{Experimental results and discussion.}

3.1 SiMULTANEOUS STM/SNOM IMAGES. - Using the commercial STM system we have obtained images in the transmission and scattering configurations. Figure 4 corresponds to the scanning of sample 2 using the transmission mode. The grating period is $200 \mathrm{~nm}$ and the scan region approximately $1.5 \mu \mathrm{m}$ by $1.5 \mu \mathrm{m}$. The STM image (left side of the figure) was taken in constant current mode with $0.42 \mathrm{mV}$ bias and $2 \mathrm{nA}$ set tunneling current. In order to obtain good optical contrast, the scan rate for electrical and optical acquisition was kept relatively slow $(<1 \mathrm{~Hz})$. 


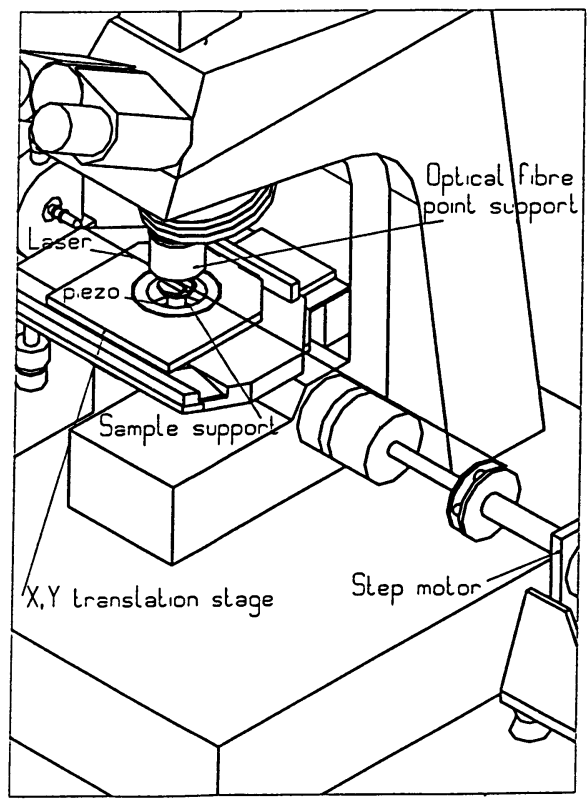

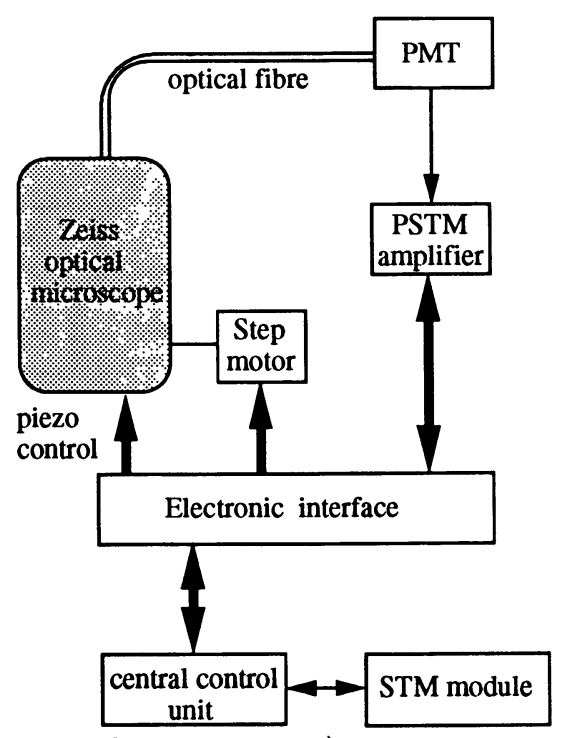

c)

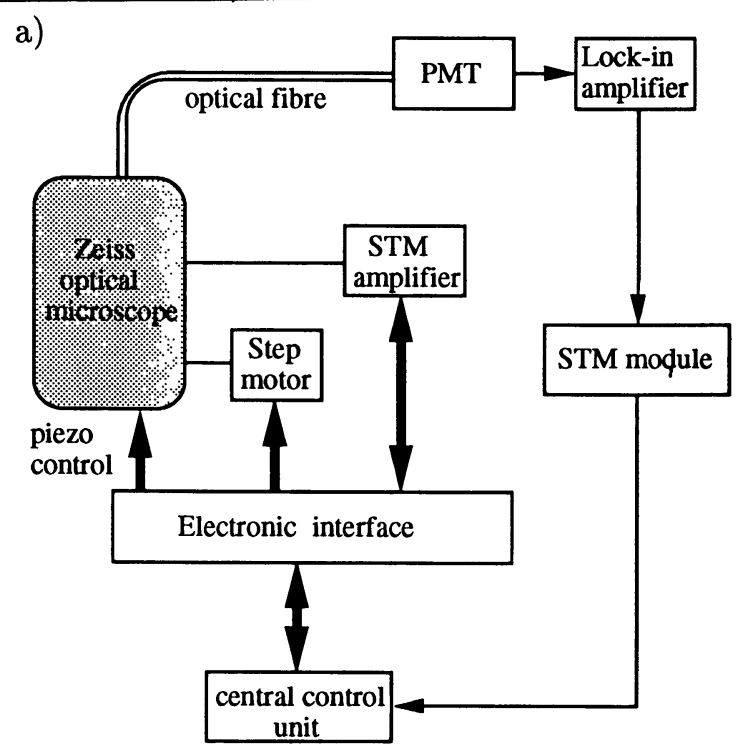

b)

Fig. 3. - Schematic diagram of the multi-task home-made microscope. Adapted Zeiss optical microscope (a), for STM regulation and imaging (b), and for PSTM measurements (c).

Faster scan rates can be used if a higher sensitive detection system is implemented (i.e. photon counting technique). One can see good resolution for both STM and SNOM images. The SNOM image (right side of the figure) shows less details; but still clear defined structures. The resolution is better than $50 \mathrm{~nm}$. The non-homogeneity in brightness observed in the SNOM image is probably due to residues of the lift-off during the fabrication of the sample. These non-homogeneities have also been observed by others [14] and explained in terms of changes of the refractive index due to the lack of smoothness on developed patterns of PMMA coated samples. Figure 5 corresponds to sample 1 using the scattering excitation mode. In spite of the fact that we have not optimised the 


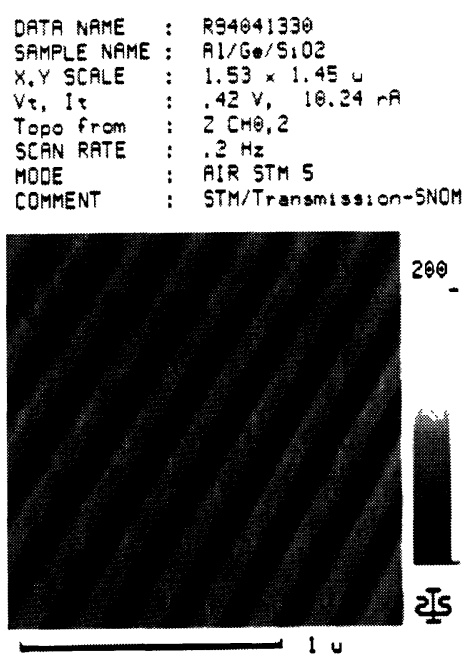

a)

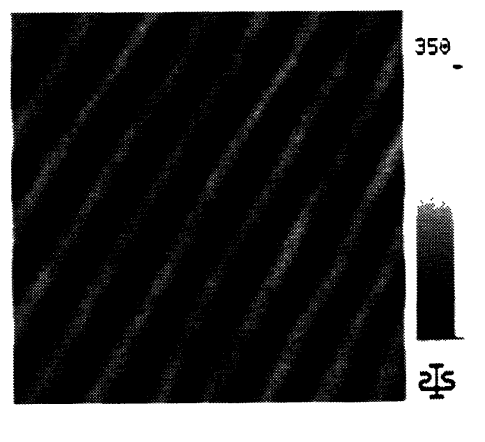

b)

Fig. 4. - Images of a simultaneous STM (a) and collection mode-SNOM in the transmission excitation configuration (b) with a $1.5 \times 1.5 \mu$ m scanning over a $200 \mathrm{~nm}$ pitch grating of the $\mathrm{Al} / \mathrm{Ge} / \mathrm{SiO}_{2}$ sample.

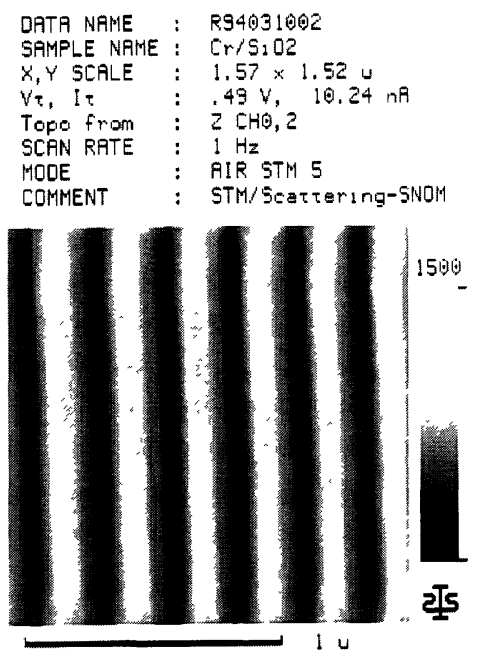

a)

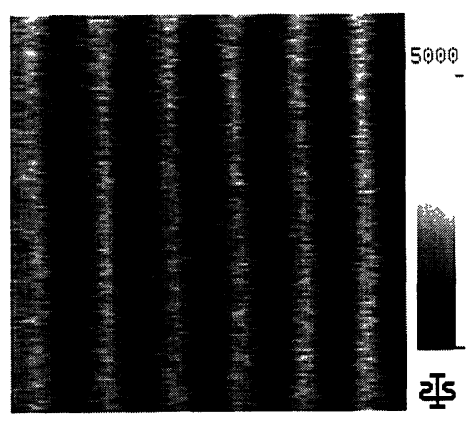

b)

Fig. 5. - Images of a simultaneous STM (a) and collection mode-SNOM in the scattering excitation configuration (b) with a $1.5 \times 1.5 \mu \mathrm{m}$ scanning over a $200 \mathrm{~nm}$ pitch grating of the $\mathrm{Cr} / \mathrm{SiO}_{2}$ sample.

angle of the incident light onto the sample, we can still resolve the trenches. However, in this case (compared with transmission), the sensitivity of the lock-in was increased and further refinements of the raw image were necessary.

The performance of a combined STM/SNOM system can be partially assessed from the above figures. In principle, high spatial-frequency components of the electromagnetic field -which contain information of the small details of the sample surface, decrease rapidly when the tip-sample separation increases. As it has been calculated [15, 16], to resolve a grating structure of period 
less than $100 \mathrm{~nm}$, the tip must be as near as $1 \mathrm{~nm}$ close to the surface. This obviously makes the tunneling regulation as one of the most powerful methods because of the sub-nanometric tip-tosample separation. Also, because of this very short separation the optical contrast is improved. In our case, the optical signal is essentially ruled by the transmission properties of the grating layer, and only local scattering affects the resultant signal.

Of course, to obtain a high resolution image, the tip aperture has to be also as small as possible. However, there is a trade-off between resolution -which requires very small aperture diameters, and intensity of the collected light required to generate an image in a reasonable amount of time. We have used single mode optical fibres with a fabrication process essentially similar to the one developed by Betzig et al. [1], except that a gold coating was used for tunneling reasons. We have found that both the shape and the aperture diameter are important for optimal guiding and maximum transmissivity. Therefore we have focused our efforts not only to achieve a small aperture, but also to control the shape, both depending on the viscosity of the glass before pulling [13].

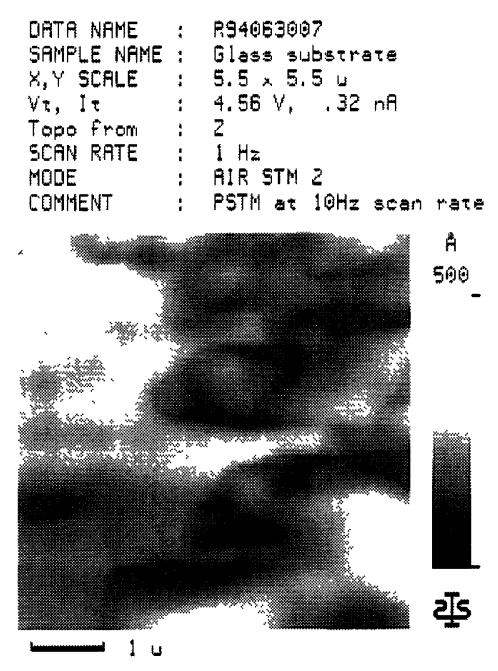

a)

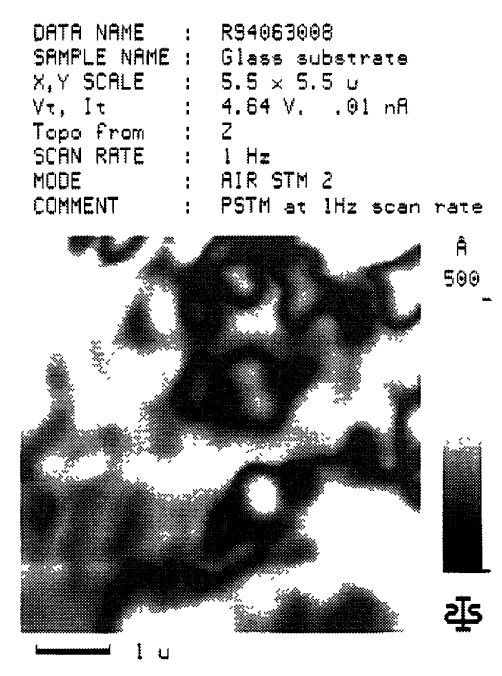

b)

Fig. 6. - PSTM images obtained on the prism surface, at $10 \mathrm{~Hz}$ scan rate (a) and at $1 \mathrm{~Hz}$ scan rate (b) with a $5.5 \times 5.5 \mu \mathrm{m}$ scan region.

3.2 FIRST RESULTS WITH A HOME-MADE SYSTEM. - So far, we have devoted our attention on the testing of the different mechanical and electronic parts that compose our system. Although we have succeeded to obtain STM images using a Pt/Ir point, and also tried simultaneous STM/SNOM measurements, we preferred -for the sake of simplicity- to test the performance of the equipment working on the PSTM configuration. For that, we simply used a dielectric optical fibre point without metallic coating and a triangle prism as the substrate to scan. Light from a He-Ne laser at $\lambda=632.8 \mathrm{~nm}$ was directed and focused to the prism at total internal reflection. For the approach of the tip to the sample and the acquisition of the optical signal we used the commercial STM module and the central control unit. Initially, we fixed a relatively large bias voltage, $4.5 \mathrm{~V}$ and a reference current of $6 \mathrm{nA}$ on the STM module. The detected optical signal was converted to current and sent to the STM module where it was compared with the reference current. If the 
detected current is smaller than the reference one (tip away from the sample), the approach of the tip onto the sample continues. The process stops when a current equal or higher than the reference current is detected. At this stage, we adjust the scan region and perform the scanning.

Figure 6 shows the images of the glass surface at two different scan rates $(10 \mathrm{~Hz}$ on the left side and $1 \mathrm{~Hz}$ on the right side). The scan region is $5.5 \mu \mathrm{m}$ by $5.5 \mu \mathrm{m}$ approximately. Good stability and reproducibility are obvious from the images, which basically demonstrate the reliability of the system. Better defined structures are observed when the scan rate is kept below $1 \mathrm{~Hz}$. The resolution is around $100 \mathrm{~nm}$, but we are confident that with the use of optimised optical fibre points we can decrease this value.

\section{Conclusions.}

We have demonstrated the possibility of simultaneous acquisition of STM and collection modeSNOM imaging with a resolution better than $50 \mathrm{~nm}$ using a commercial STM equipment and the addition of a simple, but still relevant optical set-up. The resolution and contrast of the optical image is largely improved by the short separation distance between the probe and the sample, controlled by the tunneling current. Resolution and contrast are far superior than in the case of PSTM and comparable to SNOM using shear force regulation [9]. Large intensity and good contrast are obtained by the use of a single mode optical fibre and by the reduction of diffraction effects occurring at long distances away from the sample surface. We have not studied in any detail tip-sample interactions, but we believe they are much smaller than in the case of shear force sensing which suffers from the drawback that artificial high spatial frequencies can be generated from the vertical motions induced by the feedback mechanism. A further advantage of our technique is the independence on the angle of the incident light onto the sample as already shown in this paper with the transmission and scattering configurations.

We have also succeeded on the acquisition of images in the total internal reflection configuration using a home-made system based on a Zeiss optical microscope. We would like to optimise our home-made system with the purpose of having a multi-task instrument able to work on the PSTM and SNOM modes and using either tunneling or shear force sensing as the distance regulation mechanisms. Because of the tunneling regulation mechanism, open possibilities do exist for the application of the same technique working on the scanning plasmon near-field configuration [17], and for optical spectroscopy of semiconductor microcavities, quantum wires and dots.

\section{Acknowledgements.}

This work has been partly supported by the CEE (Esprit BRA6536). The authors would like to thank H. Launois for constant interest and encouragement, E. Cambril and C. Mayeux for the technical assistance, and C. Licoppe at CNET (Bagneux) for the use of their micropipette puller. We have largely benefit from the discussions with T. Tate at Imperial College (London), on the fabrication process of the points.

\section{References}

[1] Betzig E., Trautman J., Harris T., Weiner J., Kostelak R., Science 251 (1991) 148.

[2] Durig U., Pohl D., Rohner F., J. Appl. Phys. 59 (1986) 318.

[3] Lewis A., Lieberman K., Nature 354 (1991) 24. 
[4] Harootunian A., Betzig E., Isaacso M.n, Lewis A., Appl. Phys. Lett. 49 (1986) 64.

[5] Pohl D., Denk W., M. Lanz, Appl. Phys. Lett. 44 (1984) 62.

[6] Betzig E., Isaacson M., Lewis A., Appl. Phys. Lett. 51 (1987) 208.

[7] Durig U., Pohl D., Rohner F., IBM J. Res. Develop. 30 (1986) 48.

[8] Lieberman K., Lewis A., Appl. Phys. Lett. 62 (1993) 135.

[9] Betzig E., Trautman J., Science 257 (1992) 19.

[10] Toledo-Crow R., Yang P., Chen Y., Vaez-Iravani M., Appl. Phys. Lett. 60 (1992) 2957.

[11] Garcia-Parajo M., Cambril E., Chen Y., Appl. Phys. Lett. 65 (1994) 1498.

[12] Park Scientific Instruments, 1171 Borregas Ave. Sunnyvale, CA94089

[13] Garcia-Parajo M., Tate T., Chen Y., submitted to Appl. Phys. Lett.

[14] Trautman J., Betzig E., Weiner J., DiGiovani D., Harris T., Helman F., Gyorgy E., J. Appl. Phys. Lett. 71 (1992) 469.

[15] Van Lebeke D., Barchiesi D., J. Opt. Soc. Am. A9 (1992) 72.

[16] Chen Y., Kupka R., Ultramicroscopy (1994), in press.

[17] Specht M., Pedarnig J., Heckl W., Hansch T., Phys. Rev. Lett. 68 (1992) 476. 